\title{
Gaia promises for the reference frame
}

\author{
F. Mignard \\ University Nice Sophia Antipolis, CNRS, OCA, Le Mont Gros, 06304 Nice, France
}

\begin{abstract}
Expectations of the Gaia astrometry mission regarding the realisation of an optical kinematical reference frame based on extragalactic sources are summarized.
\end{abstract}

Keywords. astrometry, reference systems

The ESA space astrometry mission Gaia is due for launch before the end of 2013. It will survey the sky over a period of 5 years with a sensitivity limited instrument down to the 20th magnitude. The astrometric accuracy measured by the quality of the parallaxes is expected to be of 25 muas at 15 mag. Gaia will simultaneously perform multi-epoch photometry for all the sources and spectroscopy to 17th mag. The mission is optimised to observe stellar sources to produce a stereoscopic and kinematic census of about one billion stars in our Galaxy enabling to probe the formation and evolution of the Milky Way. The expected astrometric accuracy in position, annual proper motion and parallaxes is shown in Fig. 1 as a function of the $G$ magnitude (very similar to $R$ band).

The scientific core of the mission is dedicated to stellar and galactic physics, and will bring considerable high quality data for these fields from the combination of the kinematical information allowed by astrometry and the low resolution spectroscopy more or less equivalent to photometry over the visible range with at least 15 bands. Each star will be observed about 70 times during the mission, with a slight scatter about this mean as a function of the ecliptic latitude. An average sequence of observations will give two successive observations separated by just above $1.7 \mathrm{~h}$, followed by similar pairs of observations every 40 to 60 days, providing a good time sampling over the mission length of 5 years. This is required to properly sample the parallactic ellipse for disentangling the proper motion and parallax, but also for investigating more complex astrometric displacement in multiple systems or for solar system objects. A full reward of this sampling will show up also with the photometric variability analysis of at least 100 millions sources.

The internal detection system will allow also to observe measure the position of several 100000 s quasars, everywhere in sky except a small area at low galactic latitude. These consistent and repeated observations will lead to the realisation of a kinematically defined inertial frame in the optical wavelengths. Given the fact that the vast majority of these QSOs will be new we have developed a procedure to recognize the QSOs from the stars in an automatic and efficient way using the photometric and astrometric information. The survey mode will aim at identifying most of the QSOs, even if it means including a certain fraction of stellar contaminant in the set. In parallel a more restrictive setting of the selection parameters will enable to generate a clean sample of sources, incomplete but free of contaminant, to serve as defining sources for the establishment of the inertial frame.

From simulations based on realistic space density of optical QSOs as a function of magnitude and using the Gaia astrometric performance, Gaia should realise a quasiinertial celestial reference frame with a residual rotation better than $0.3 \mu$ as per year. This is illustrated in Fig. 2 giving on the left scale the expected accuracy as a function 


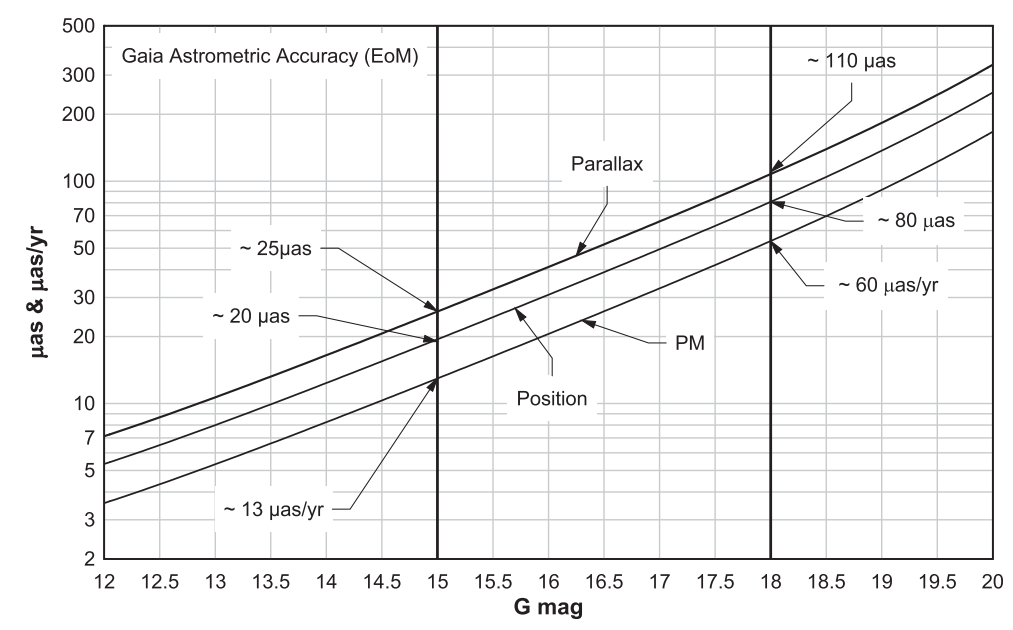

Figure 1. End-of-Mission astrometric accuracy expected with Gaia for the position, proper-motion and parallaxes for stars and extragalactic point-like sources.

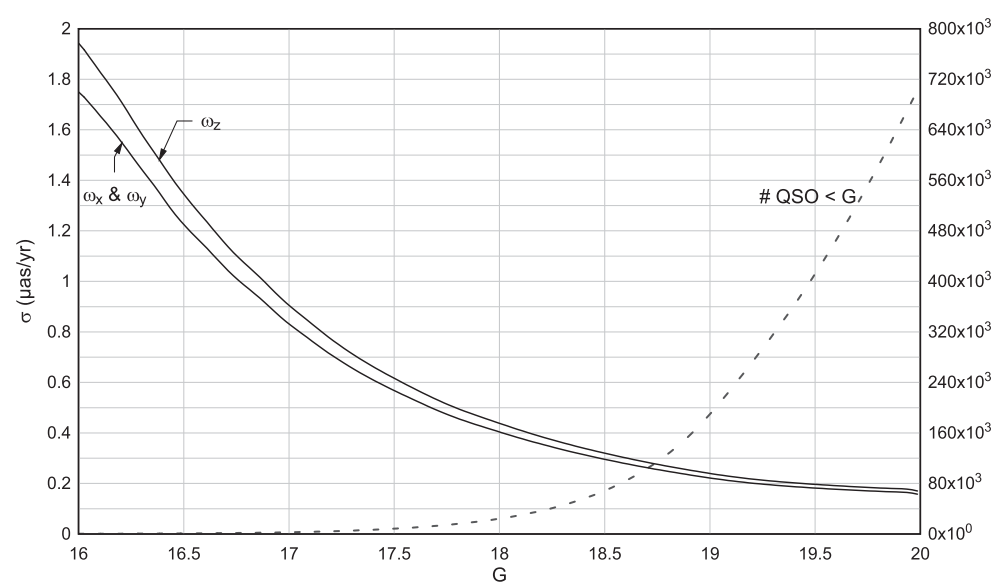

Figure 2. Inertiality of the final Gaia Celestial Reference Frame based on the QSO observations, expressed by the accuracy of the residual rotation in galactic coordinates. The precision for a $G$ magnitude is computed with only sources brighter than $G$. A random instability has been taken equal to $20 \mu \mathrm{as} \mathrm{yr}^{-1}$ and added (quadratic sum) to the single star noise. The right scale gives the number of sources found brighter than $G$.

of the magnitude of the faintest sources kept in the sample. The number of sources is shown on the dashed line read on the right scale. Eventually the final Gaia product directly related to the Celestial Frame will include also all the stellar sources given in the same system with accurate proper motions. The whole set should be referred to as the Gaia-CRF (Gaia Celestial Reference Frame) which will comprise:

- A set of defining sources from the clean subset of QSOs used to fix the frame spin

- A larger set of secondary QSOs not used to remove the residual rotation

- A very small set of QSOs common to Gaia and ICRF2 used to tie the orientation

- The Gaia-SRF (Stellar Reference Frame):

a. about one billion stars with positions, proper motions and parallaxes;

b. an average density of $\sim 25000$ stars per square degree, highly variable with galactic latitude. 